\title{
ENGLISCH MEAD, POLNISCH MIÓD UND HOCHDEUTSCH MET AUS DER HISTORISCH-VERGLEICHENDEN PERSPEKTIVE
}

\section{Einführung}

Die diachronische Analyse, die im vorliegenden Artikel durchgeführt wird, hat es sich zur Aufgabe gestellt, eine neue Herangehensweise zu den vergleichenden Untersuchungen zu veranschaulichen (Rychło 2019). Normalerweise berücksichtigt die kontrastive Linguistik eine diachronische Perspektive nicht (vgl. Hellinger 1977, S. 2), aber sie beabsichtigt Gemeinsamkeiten und Unterschiede zwischen zwei (oder mehreren) Sprachen zu beschreiben und hoffentlich auch zu verstehen. Wenn die Sprachen jedoch verwandt sind, ist ein bedeutender Teil der Gemeinsamkeiten dadurch verursacht, dass sie von einer gemeinsamen Ursprache abstammen. Ebenso liegen die Gründe für Unterschiede in den Veränderungen, die sich auf die verwandten Sprachen über die Jahre hinaus auswirkten, zumindest in einem gewissen Maß. Deshalb ist die These, die die vorliegende Herangehensweise charakterisiert, eine solche, die in der diachronischen Perspektive eine Vorbedingung für das Verstehen der Gemeinsamkeiten und Unterschiede zwischen verwandten Sprachen sieht und demzufolge sollten die Untersuchungen des Wandels, dem die zu vergleichenden Sprachen unterliegen, zu einem großen Teil der kontrastiven Sprachwissenschaft werden.

Das Beispiel, das in der vorliegenden Arbeit untersucht wird, ist ein klarer und unumstrittener Fall der genau verwandten Wörter, d. h. der Vorfahr des poln. miód hat dieselbe Urform, wie die von englischem mead und dem deutschen Met. Das Projekt, beschrieben weiter oben und entwickelt in Rychło (2012, 2013, 2014a, 2014b, 2016, 2017, 2018), hat es sich zur Aufgabe gestellt, die älteste Schicht in der polnischen und englischen Gegenwartssprache auszumachen und zu untersuchen. Der vorliegende Beitrag berücksichtigt ausnahmsweise auch den hochdeutschen Met, weil die Form das Verstehen der Veränderungen erleichtert.

* Dr. Mikołaj Rychło, Universität Gdańsk, Institut für Anglistik und Amerikanistik, Wita-Stwosza 51, 80-308 Gdańsk. E-Mail: mrychlo@wp.pl 


\section{Der Beweis}

Der erste Schritt bei der Untersuchung ist, sich zu vergewissern, ob die analysierten Wörter seit den frühesten Perioden in der Geschichte der beiden Sprachen belegt sind. Dieser Test bestätigt, dass miód seit dem 14. Jh. im Polnischen anwesend ist - das Wort ist in Stownik staropolski (Nitsch, Klemensiewicz und Urbańczyk 1953-2002, Band 4, S. 281) verzeichnet. Das hochdeutsche Wort Met ist seit dem 9. Jh. belegt (cf. Kluge-Seebold 2011, S. 618). Auch das Wort mead kommt seit Beowulf im Englischen vor. Es befindet sich an verschiedenen Stellen, unter anderem gleich in den Anfangsstrophen, im Kompositum meodosetla (Gen. Pl.) von meodosetl ,Metsitz':

Oft Scyld Scéfing sceapena préatum

(Der Garbensohn Scyld hat oft grimme Feinde,)

monegum maégpum meodosetla oftéah

(Viel mutige Krieger vom Metsitz verjagt)

Der zweite Schritt besteht darin, die verwandten Wörter aus den nächstliegenden Sprachen zu sammeln und ihre Reichweite zu erkunden. Dadurch wird möglich sein, das Alter des gemeinsamen Vorfahren zu bestimmen.

Polnisch miód ist ein Grundwort für ,Honig' und ist verwandt mit akslaw. medъ ,Honig', russ. mёd (мёд) ,Honig, Met', ukr. mid „Honig' G. médu, wruss. med ,Honig', bulg. med „Honig'skr. mêd „Honig' G. mëda, sloven. mêd „Honig', čech. slk. med „Honig', osorb. měd „Honig', nsorb. mjod „Honig' (cf. Vasmer 1953-1958, S. 110; Derksen 2008, S. 306-307).

Die germanischen verwandten Wörter sind nicht das englische honey und das deutsche Honig, sondern engl. mead, und deutsch Met, die ein alkoholisches Getränk aus vergorenem Honig, oder ,Honigwein ‘ bezeichnen. In alten germanischen Sprachen nimmt das Wort folgende Formen an: ahd. metu, meto (seit dem 9. Jh.), mhd. met(e), mnd. mnl. afries. nl. mede, aengl. medu, meodu, anord. mjoðr, isl. mjöður, färöisch mjøður; norw. mjød; dän. mjød, schwed. mjöd. Aufgrund dieser Formen ist die urgermanische Rekonstruktion unumstritten und sie sieht folgend aus: *medu- (cf. Onions 1966, S. 564; Magnússon 1989, S. 627; Kluge-Seebold 2011, S. 618; Kroonen 2013, S. 361).

Das Wort für ,Met' ist in vielen alten Sprachen belegt, insbesondere: aind. mádhu- ,süßer Trank, Süßigkeit, Honig, Milch', Pali madhu-, Hindi mau ,Honig', avest. $m a \delta u$ - ,Honig, Beerenwein', griech. méthy ( $\mu \varepsilon \dot{\theta} \theta$ ), Wein, berauschendes Getränk', air. mid ,Met', kymr. medd ,Met', lit. medùs ,Honig', lett. medus ,Honig, Met', apreuß. meddo, toch. B mit ,Honig'. Puhvel (1984.6, S. 100-101) führt auch an: hethitisch ${ }^{\mathrm{INDA}}$ madu ,süßes Brot' oder ,Honigbrot", luwisch maddu- ,Wein“ (Nom.- Akk. Sg. ma-ad-du); hieroglyphen-luwisch matu-, Wein‘ aus idg. *medhu- . 
Was die urindogermanische Rekonstruktion betrifft, wird ein idg. u-stammiges Neutrum angenommen, das auf die folgende Art und Weise erschlossen wird: nach Pokorny (1959, S. 707): *médhu ,Honig; bes. Met'; nach Mann (1984/87, S. 742-743): *medhu ,süßes Getränk, flüssiger Honig, Met'; nach Watkins (2011, S. 53): *medhu ,Honig', auch ,Met"; nach Gamkrelidze und Ivanov (1995.I, S. 517-518) ${ }^{1}$ : med $^{h} u$,Met, Honig'; nach Mallory und Adams (1997, S. 271) *médhu ,Met'; nach Onions (1966, S. 564) *medhu-; nach Kluge-Seebold (2011, S. 618) *med ${ }^{h} u$-; nach Derksen (2008, S. 306-307): *med $u$.

Es ist bemerkenswert, dass es auch sehr ähnliche Formen in außerindogermanischen Sprachen gibt, insbesondere in der uralischen Sprachfamilie, in der finnisch-ugrischen Gruppe: finnisch mesi (Gen. meden) ,Honig, Met', estnisch mesi (Gen. mee) ,Honig', erzä-mordwinisch med' mäd', mokscha-mordwinisch med', ?tscheremissisch mü, müj, wotjakisch $m u, m u$, syrjänisch $m a$,Honig'; ungarisch méz „Honig‘. Aufgrund dieser Formen, Rédei (1986-1988, S. 273) rekonstruiert finnisch-ugrisch * mete ,Honig, aus Honig gegorener Trank und es wird als eine indogermanisch Entlehnung behandelt.

Ähnliche Benennungen für ,Honig' sind im Etruskischen belegt: $m a \theta$,Honig, Honigwein', und in den drawidischen Sprachen: Tamil mațtu, Honig, Toddy, gegorener Trank, süßer Saft, Getränk, das während der sexuellen Vereinigung getrunken wird, Likör, Wohlgeruch', mațtam ,Toddy', Malayalam mațu ,Süße, Honig', matțu, Nektar' und Tulu mitți ,Süße', mitțè ,Pollen' - cf. Burrow und Emeneau (1984, S. 412, no. 4662). Nach Vasmer (1953-1958, S. 110, s.v. мед) treten anklingende Wörter auch im Chinesischen und Japanischen auf, vgl. urchines. *mit, japan. mitsu, deren Herkunft im idg. gesucht wird, aber die Art ihres Zusammenhangs noch nicht ausreichend geklärt ist. Es ist bemerkenswert, dass urchines. *mit aus dem Tocharischen entlehnt werden konnte (vgl. toch. B mit ,Honig').

Der Nachweis der Kontinuität, d. h. die Vergewisserung des ununterbrochenen Verlaufs der vergleichenden Wörter, als auch die Nebeneinanderstellung der verwandten Wörter gehören zu den ersten zwei Etappen und setzen sich somit auch das erste Ziel, die älteste Schicht in der polnischen und deutschen Gegenwartssprache auszumachen. Diese Schicht ist in den beiden Sprachen aufgrund ihres gemeinsamen Ursprungs präsent. Dennoch werden im Laufe der Zeit mannigfaltige Unterschiede auftreten. Deshalb erscheint es zweckmäßig, im Weiteren gerade auf diese Unterschiede näher einzugehen. Ein anderes Ziel der Herangehensweise ist, diese Unterschiede zu erklären, indem der Evolution der beiden verwandten Wörter nachgegangen und ihre Entwicklung seit dem Urindogermanischen rekonstruiert wird. Das wird zum Gegenstand der weiteren Analyse. Wenn es gelingt, alle Laute in den

${ }^{1}$ Gamkrelidze und Ivanov (1995.I: S. 517-518) liefern interessante Informationen sowohl über die Imkerei als auch den semantischen Unterschied zwischen *melit ${ }^{h}$ - und $* m e d^{h} u$ - und ihre spätere Entwicklung. 
zu vergleichenden Wörtern in einen Zusammenhang zu bringen, wobei sich die Unterschiede aus ebendiesen Klangveränderungen ergeben, wird das als Bestätigung der Verwandtschaft der analysierten Wörter betrachtet.

\section{Eine diachronische Analyse}

Als Ansatzpunkt bei der Suche nach der Erklärung von lautlichen Verschiedenheiten zwischen englisch mead und polnisch miód, fangen wir mit urindogermanischem *med ${ }^{h}$-u - an. Sowohl im Slawischen, als auch im Germanischen wurden die urindogermanischen stimmhaften aspirierten Verschlusslaute, wie $* d^{h}$ zu stimmhaften Verschlusslauten, wie $d$. Im Urgermanischen ist es auch möglich, dass $* d^{h}$ sich zuerst zu einer Frikative $* \delta$ entwickelte, bevor es sich unter gewissen Bedingungen zum stimmhaften unaspirierten Verschlusslaut $* d$ verhärtete (cf. Lass 1994, S. 76-77). Deshalb rekonstruierte Orel (2003, S. 265) das urgermanische *međuz, aber, nach Kroonen (2013, S. 361), urgermanisch *medu$\left(<* m e d^{h}-u-\right)$.

Im Deutschen veränderte sich das urgermanische $* d$ zu $t$ nach der zweiten Lautverschiebung, von daher Met. Aspirata sind im Altindischen als $d h$ bewahrt und im Altgriechischen als $\theta$. Weitere Beispiele von polnisch-englisch-deutschen verwandten Wörtern, die die gleiche Übereinstimmung zwischen den Konsonanten aufweisen (polnisch $d$, englisch $d$, deutsch $t$ ), enthalten:

polnisch wdowa, englisch widow, deutsch Witwe (vgl. aind. vidhávā) polnisch trzoda, englisch herd, ahd. herta $>$ Herde (vgl. aind. śárdha-) polnisch drzwi, englisch door, deutsch Tür (vgl. griech. $\theta 0 ́ \rho \alpha)$

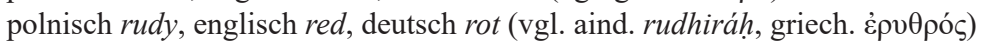

Ebenso:

polnisch miód, englisch mead, deutsch Met (vgl. aind. mádhu)

Im Polnischen wurde der Konsonant $m$ bei Vokalen der vorderen Reihe palatalisiert, was typisch für alte polnische Wörter, z. B. mieć ,haben', miejsce ,Platz', miedza ,Feldrein', mienić ,schillern', mierzyć ,ausmessen", miesiąc „Monat", mieszać ,verrühren' ist. Die Palatalisierung wurde phonologisiert nach der polnischen Vokalumlautung, die unten erwogen wurde, aber das erweichte Allofon muss früher aufgetreten sein.

Im Polnischen ist die Verbindung me fast immer die Folge der Entlehnungen, die in den meisten Fällen, in den letzten drei Jahrhunderten in der Sprache aufgetaucht sind; zum Beispiel: mebel ,Möbelstück', mecz ,Match' meczet ,Moschee', medal ,Medaille', meduza ,Meduse', meldować ,melden', melina ,Saufbude', meta ,Ziel', metal ,Metall', meteor ,Meteor', metoda „Methode', metr ,Meter', metro ,U-Bahn', metropolia ,Metropole', metresa „Mätresse', mewa „Möwe', mezalians 
„Mesalliance‘. Keines dieser Wörter war im Altpolnischen vertreten. Die wenigen früheren Entlehnungen weisen auf die Alternation zwischen me und $m a$ (und vor $n$ auch ę): melon $\sim$ malon ,Melone', mendel $\sim$ mandel $\sim$ mędel ,Mandel' hin. Das Auftreten der Kombination me in den ererbten Wörtern ist sehr selten und erscheint dann, wenn ein hinteres Jer (ъ), das sich in sogenannter starker Position befand, der Vokalisierung unterlag. Zwei Tatsachen müssen berücksichtigt werden: erstens, die vorangehenden Konsonanten wurden nicht durch ein hinteres Jer palatalisiert, und zweitens, im Polnischen war das Resultat der Vokalisierung von Jers der Vokal $e$. Diese Entwicklungen erklären, warum $e$ in poln. mech ,Moss' auftritt.

Der erweichte Konsonant (in der Schrift durch die Buchstaben $m i$ wiedergegeben) ist eine Spur davon, dass der Vokal $e$ einmal in poln. miód präsent war. Ein ähnlicher Vokal ist im deutschen Met anwesend. Eine andere Spur der ehemaligen Anwesenheit von einem Vokal der vorderen Reihe im poln. miód kann in dem Wort niedźwiedź ,Bär' gefunden werden.

Infolge der polnischen Vokalumlautung wurde $* e$ vor harten Vorderzungenkonsonanten $(t, d, n, s, z)$ und den harten liquiden Konsonanten $(r, \nmid)$ zu $o$. Infolgedessen wurde *med zu * mod $\mathbf{z}$ verändert. Immer wenn ein anderer Konsonant folgte, blieb $e$ unverändert. Infolgedessen haben wir viele Alternationen im Polnischen, wie zum Beispiel niose, ich trage' : niesie, er trägt', wioze, ich befördere' : wiezie, er befördert ${ }^{\star}$. Der Grund, warum $e$ erhalten bleibt ist es, dass ein palatalisierter Konsonant, nach dem $e$ folgte, wie in niesie, wiezie (in der Schrift durch die Digrafen $<\mathrm{si}>,<\mathrm{zi}>$ wiedergegeben. Ebenso blieb in dem Wort niedźwiedź ein unveränderter Vokal $e$ vor erweichtem $d z$.

Der nächste Lautwandel - der Ausfall von Jers - hatte eine Ersatzdehnung zur Folge, d. h. nach dem Wegfall des Jers wurde der vorausgehende kurze Vokal „ersatzweise“ gedehnt, so dass ḿodъ wurde zu mo: $d$, und gleichzeitig, als $d$ fand sich in Auslaut, wurde es stimmlos. Nächstens wurden der lange Vokal $o$ : zu $u$ verengt, wodurch die phonologische Quantität in die Qualität überging. Im Genitiv sg. gab es die $u$-Endung anstatt $\mathrm{b}$ und, folglich, gab es keine Ersatzdehnung und keine Verengung. Infolgedessen sind im Polnischen viele Alternationen erschienen, wie miód : miodu „Honig', róg : rogu „Horn', wór : wora ,Sack', lód : lodu ,Eis', dwór : dworu ,Hof', dziób : dziobu ,Schnabel'. Auch in Verben kann man ähnliche Effekte beobachten, z. B. nióst, er trug': niosła ,sie trug', wiózt, er trug': wiozła, sie trug'.

Im Englischen sind zwei Veränderungen für die Aussprache /i:/ in mead verantwortlich: Frühneuenglische Vokalverschiebung und die Mittelenglische Dehnung in offener Silbe. Nach der Vokalverschiebung: / $\varepsilon$ :/ > e:/ > /i:/, aber der Wandel hätte sich nicht ereignet, wenn der Vokal kurz gewesen wäre. Deshalb musste eine andere Veränderung vorgehen, die den Vokal gedehnt hatte. Dieser Wandel fand in offener Silbe zweisilbiger Formen statt, wie aengl. medu. Infolgedessen wurde der altenglische kurze /e/ zu frühmittelenglische / $\varepsilon: /$, die in der Schrift durch die Buchstaben $e a$ wiedergegebenen wird (im Mittelenglischen gab es auch 
/e:/, durch die Buchstaben ee wiedergegebenen, die endlich mit /ع:/ zusammengelaufen ist;, daher viele Homofone, z. B. sea ,Meer : see, sehen', weak, schwach : week,Woche', meat ,Fleisch': meet ,treffen', steal ,stehlen' : steel ,Stahl'). Das Deutsche bewahrt den Vokal verhältnismäßig unverändert, vgl. Met.

\section{4. * medvědb}

Das urslaw. Wort *medz (poln. miód) befindet sich auch als ein von zwei Wurzeln in Nomen Compositum *medvědb (poln. niedźwiedź) ,Bär', der kann

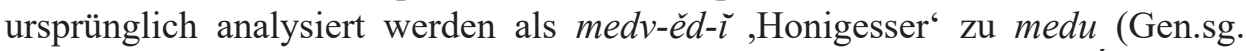
von medъ) und *ěd ein deverbaler Nomen von urslaw. *ěsti (poln. jeść) ,essen“. Die Entwicklung kann rekonstruiert werden, wie im Folgenden: *medu-éd $\mathbf{b}>$ *meduédb > *medvědb - cf. Boryś (2005, S. 360-361).

Eine mögliche Erklärung von poln. $n i$ in niedźwiedź wäre, dass es zu einer Dissimilation gekommen ist, so dass $m i$ zu $n i$ wurde, um das labiale Merkmal von wi in der zweiten Silbe zu unterscheiden. Eine andere Erklärung wäre, dass $n$ durch eine Fernassimilation an $d$ und eine Anlehnung an $n e$ - entstanden ist - cf. Vasmer (1953-1958, S. 110, s. v. медве́дь).

Die übrigen slawischen verwandten Wörter bewahren das ursprüngliche $m$, nur im Tschechischen ist eine Form mit $n$ belegt: akslaw. medvědb, russ. medvéd', ukr. médvid', védmid', skr. mèdvjed, sloven. médvẹd, čech. medvěd, nedvěd, dial. miedźwiedź, osorb. mjedwjédź, nsorb. mjadwjeź - cf. Derksen (2008, S. 306); Vasmer (1953-1958, S. 110, s.v. медве́дь).

$\mathrm{Zu}$ beachten ist, dass das Wort nicht nur im Slawischen belegt ist, sondern auch im Aind. madhv-ád- m. ,Honigesser, Süßes essend“ - cf. RV 1,164,22 madhuv-ádah suparnậh , die Honigsüßigkeit essenden Vögel‘ (Mayrhofer 1992, S. 302).

Die Anwesenheit eines solchen Kompositums kann dadurch erklärt werden, dass ein Tabu den Schwund der indogermanischen ursprünglichen Bezeichnung

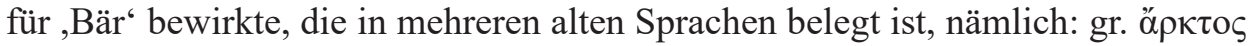
f. ,Bär', lat. ursus ,Bär', aind. 'rkșa- m., jav. arša- m. ,Bär', arm. arǰ ,Bär' (nach Pokorny 1959, S. 875, beeinflusst von arjn, dunkelbraun'), alb. ari, mir. art, kymr. arth, hethitisch hartagga- ,Bär' oder ,Wolf', etc. Die urindogermanische Rekonstruktion ist *'kkpo-s (Pokorny 1959, S. 875), *rkpos (Vasmer 1953-58, S. 110, s. v. медве́дь), * $h_{2} r t \hat{k}$ - (cf. Beekes 2009, S. 133; Martirosyan 2009, S. 143).

\section{Schlussfolgerungen}

Polnisch und Englisch, wie alle lebenden Sprachen, haben im Laufe ihrer Geschichte bekanntlich eine große Anzahl von Veränderungen durchgemacht. Diese Veränderungen haben zu einem deutlichen Wandel in der Gesamtstruktur der 
Sprachen geführt. Das polnische Wort hat sich aus idg. ${ }^{*} m e d^{h}-u$ - entwickelt und die folgenden Veränderungen erfahren.

$\begin{array}{ll}\text { med }^{h}-u- & \\ \text { medu- } & \text { Deaspiration } \\ \text { med } \mathrm{b} & \text { Entwicklung von Jers } \\ \text { med } \mathrm{b} & \text { Palatalisierung } \\ \text { modb } & \text { polnische Vokalumlautung } \\ \text { ḿo:d } & \text { Ausfall von Jers und } \\ \text { ḿo:t } & \text { Ersatzdehnung } \\ \text { mut } & \text { Hebung }\end{array}$

Das englische Wort stammt aus der gleichen Proto-form und unterlag den folgenden Verwandlungen:
med ${ }^{h}-u-$
medu Deaspiration (erste Lautverschiebung, Rask-Grimm-Gesetz)
medo die Abschwächung unbetonter Silben
$m \varepsilon: d a \quad$ Mittelenglische Dehnung in offener Silbe
me:d Frühneuenglische Vokalverschiebung und Apokope
mi:d Hebung

Das hochdeutsche Wort hat folgende Verwandlungen durchgemacht.

$\begin{array}{ll}\text { med }^{\text {h}}-u- & \\ \text { medu } & \text { Deaspiration (erste Lautverschiebung, Rask-Grimm-Gesetz) } \\ \text { metu } & \text { zweite Lautverschiebung } \\ \text { metə } & \text { die Abschwächung unbetonter Silben } \\ \text { me:tə } & \text { Dehnung in offener Silbe } \\ \text { me:t } & \text { Apokope }\end{array}$

Es gibt keine Zweifel, dass die drei Wörter verwandt sind. Im vorliegenden Beitrag wurde ein Versuch unternommen, die Unterschiede zwischen dem polnischen miód dem englischen mead und dem hochdeutschen Met zu erklären. Gleichzeitig haben wir es mit einem archaischen Relikt zu tun, das zeigt, dass unsere Vorfahren Met kannten und das Wort ein Teil des gemeinsamen Sprachschatzes war.

\section{LITERATURVERZEICHNIS}

Beekes R. (2009), Etymological Dictionary of Greek (with the assistance of Lucien van Beek), Leiden: Brill.

Beowulf (1906), Text mit diakritischen Zeichen, mit paralleler Übersetzung von Hugo Gering, bearbeitet von Benjamin Slade, British Library MS Cotton Vitellius A.xv (Gneuss 399), http:// www.heorot.dk/beo-intro-de.html (Zugriff am: 19.12.2018). 
Boryś W. (2005), Słownik etymologiczny języka polskiego, Kraków: Wydawnictwo Literackie.

Burrow T., Emeneau M. (1984), A Dravidian Etymological Dictionary, 2. Aufl., Oxford: Clarendon Press. Derksen R. (2008), Etymological Dictionary of the Slavic Inherited Lexicon, Leiden-Boston: Brill. Gamkrelidze T., Ivanov V. (1995), Indo-European and the Indo-Europeans, Berlin: Mouton de Gruyter. Hellinger M. (1977), Kontrastive Grammatik Deutsch/Englisch, Tübingen: Niemeyer.

Kluge F., Seebold E. (2011), Kluge Etymologisches Wörterbuch der deutschen Sprache, 25. Aufl. Berlin-Boston: De Gruyter.

Kroonen G. (2013), Etymological Dictionary of Proto-Germanic, Leiden-Boston: Brill.

Lass R. (1994), Old English: A historical linguistic companion, Cambridge: Cambridge University Press. Magnússon Á. (1989), Icelandic Etymological Dictionary, Orðabók Háskólans.

Mallory J., Adams D. (Hrsg.) (1997), Encyclopedia of Indo-European Culture, London-Chicago: Fitzroy Dearborn.

Mann S. (1984), An Indo-European Comparative Dictionary, Hamburg: Helmut Buske.

Martirosyan H. (2009), Etymological Dictionary of the Armenian Inherited Lexicon, (Leiden Indo-European Etymological Dictionary Series, vol. 8.), Leiden-Boston: Brill.

Mayrhofer M. (1992), Etymologisches Wörterbuch des Altindoarischen, Heidelberg: Carl Winter - Universitätsverlag.

Nitsch K., Klemensiewicz Z., Urbańczyk S. (Hrsg.) (1953-2002), Stownik staropolski, Warszawa: Polska Akademia Nauk.

OED The Oxford English Dictionary, 3. Aufl.: OED Online, März 2000-, Hg. John A. Simpson, http://www.oed.com (Zugriff am: 19.12.2018).

Onions C. (1966), The Oxford Dictionary of English Etymology, Oxford: Oxford University Press.

Orel V. (2003), A Handbook of Germanic Etymology, Leiden/Boston: Brill.

Pokorny J. (1959), Indogermanisches Etymologisches Wörterbuch, Bern und Munchen: Francke Verlag.

Puhvel J. (1984-1997), HED Hittite Etymological Dictionary, I-IV, Berlin-New York-Amsterdam: Mouton de Gruyter.

Rédei K. (Hg.) (1988), Uralisches Etymologisches Wörterbuch, Budapest: Akadémiai Kiadó.

Rychło M. (2012), An etymological comparison of English 'fist' and Polish 'pięśc': How they both descended from the PIE root for 'five'. In: Linguistica Silesiana 33, S. 19-38.

Rychło M. (2013), English 'herd' and Polish 'trzoda': How the two words developed from one Proto-Indo-European root. In: Acta Neophilologica XV (1), S. 155-166.

Rychło M. (2014a), Ślady prawa Grimma w angielszczyźnie w zestawieniu z polskimi wyrazami pokrewnymi: pie. ${ }^{*}$ p > pgerm. *f. In: Język Polski XCIV (3), S. 200-211.

Rychło M. (2014b), Przejście pie. *p w pgerm. *f utrwalone w polsko-angielskich wyrazach pokrewnych: praformy różniace się budowa słowotwórcza oraz późniejsze zmiany modyfikujace skutki prawa Grimma. In: Język Polski XCIV (5), S. 452-462.

Rychło M. (2016), Can weep lure? An analysis of a controversial Slavico-Germanic pair of cognates, In: Beyond Philology 13, S. 103-124.

Rychło M. (2017), Kontrowersyjne ślady zmiany pie. *p > pgerm. *f utrwalone w polsko-angielskich wyrazach pokrewnych: zestawienia sporne $i$ wczesne zapożyczenia. In: Język Polski XCVII (3), S. 114-128.

Rychło M. (2018), Polish 'reż' and English 'rye' concealed in Polish 'rżysko': A case study of a pair of cognates and a root archaism. In: Linguistica Silesiana 39, S. 119-133.

Rychło M. (2019), Contrasting Cognates in Modern Languages from a Diachronic Perspective, Gdańsk: Wydawnictwo Uniwersytetu Gdańskiego.

Vasmer M. (1953-1958), Russisches Etymologisches Wörterbuch, Heidelberg: Carl Winter.

Watkins C. (2011), The American Heritage Dictionary of Indo-European Roots, 3rd Edn., New York: Houghton Mifflin Harcourt, Boston. 
Mikołaj Rychło

\section{ENGLISH MEAD, POLISH MIÓD AND GERMAN MET FROM A HISTORICAL AND COMPARATIVE PERSPECTIVE}

(Summary)

The paper presents a diachronic analysis of Polish miód 'honey', English mead and German Met 'mead' conducted according to a new approach to contrastive studies. Taking into account potential cognates in other languages, the work aims to investigate the evolution of the common ancestor word *med ${ }^{h}-u$ - in two lines of development: the Slavic leading to modern Polish, and the Germanic leading to modern English and High German. In order to understand these branching paths, the pertinent sound changes have been identified, which transformed the common proto-form. These developments are illustrated with further examples and, in the summary, ordered chronologically. The paper also discusses an old compound *medvédb (Polish niedźwiedź), which can be considered a taboo designation for a bear, and contains also the root *meds 'honey'.

Keywords: Slavic-Germanic cognates, diachronic analysis, sound change, contrastive analysis, relic 